\title{
Pseudo-Finsler spaces modeled on a pseudo-Minkowski space
}

\author{
A. García-Parrado Gómez-Lobo \\ Física Teórica, Universidad del País Vasco, \\ Apartado 644, 48080 Bilbao, Spain. \\ e-mail: alfonso@math.uminho.pt \\ E. Minguzzi \\ Dipartimento di Matematica e Informatica "U. Dini", \\ Università degli Studi di Firenze, \\ Via S. Marta 3, I-50139 Firenze, Italy. \\ e-mail: ettore.minguzzi@unifi.it
}

April 10, 2018

\begin{abstract}
We adopt a vierbein formalism to study pseudo-Finsler spaces modeled on a pseudo-Minkowski space. We show that it is possible to obtain closed expressions for most of the geometric objects of the theory, including Berwald's curvature, Landsberg's tensor, Douglas' curvature, non-linear connection and Ricci scalar. These expressions are particularly convenient in computations since they factor the dependence on the base and the fiber. As an illustration, we study Lorentz-Finsler spaces modeled on the Bogoslovsky Lorentz-Minkowski space, and give sufficient conditions which guarantee the Berwald property. We then specialize to a recently proposed Finslerian pp-wave metric. Finally, the paper points out that non-trivial Berwald spaces have necessarily indicatrices which admit some non-trivial linear group of symmetries.
\end{abstract}

Keywords: Finsler geometry, Minkowski space, Berwald space.

\section{Introduction}

In this work by pseudo-Minkowski space we mean a pair $(V, L)$ where $V$ is an $m$-dimensional vector space and $L: \bar{\omega} \rightarrow \mathbb{R}$ is a function defined on the closure of an open cone $\Theta^{1} \omega \subset V$ with vertex the origin, such that

(a) $L \in C^{4}(\omega) \cap C^{0}(\bar{\omega})$,

\footnotetext{
${ }^{1}$ We do not assume it to be convex.
} 
(b) $\forall s>0$, and $y \in V$ we have $L(s y)=s^{2} L(y)$,

(c) the Hessian $g:=\left(\partial_{\mu} \partial_{\nu} L\right) \mathrm{d} y^{\mu} \otimes \mathrm{d} y^{\nu}$ is non-degenerate on $\omega$.

It is understood that the Hessian is calculated using any set of linear coordinates on $V$ (namely a dual basis on $V^{*}$ ). More specific theories are possible, for instance if $g$ is positive definite and $\omega=V \backslash 0$ one speaks of Minkowski space, while if $g$ has Lorentzian signature and $\omega$ is a sharp convex cone one speaks of Lorentz-Minkowski space. We warn the reader that already in the positive definite case the concept of Minkowski space is not homogeneously defined in the literature (compare [1, 2]).

If we were concerned with just Minkowski and Lorentz-Minkowski spaces we would probably add the condition

(d) $\left.L\right|_{\omega} \neq 0$ and $\left.L\right|_{\partial \omega}=0$,

in the definition. However, we shall not use it in our derivations (so vector spaces endowed with a non-degenerate bilinear form are included in the definition).

A pseudo-Finsler space is instead a pair $(M, \mathscr{L})$ where $M$ is a $m$-dimensional manifold and $\mathscr{L}: \bar{\Omega} \rightarrow \mathbb{R}, \Omega \subset T M, \pi(\Omega)=M$, with the property that if we define $\Omega_{x}:=\pi^{-1}(x) \cap \Omega, \mathscr{L}_{x}:=\mathscr{L}_{\bar{\Omega}_{x}}, \Omega_{x} \subset T_{x} M$, then the pair $\left(T_{x} M, \mathscr{L}_{x}\right)$ is a pseudo-Minkowski space with a signature independent of $x$. One can assume various differentiability conditions on the dependence of $\mathscr{L}$ on $x$, for our calculations $C^{2}$ will suffice. Of course, one could define Finsler and LorentzFinsler spaces adding the conditions introduced above for pseudo-Minkowski spaces.

Remark 1. The domain of $L$ is really of little importance for the purpose of this work since all our calculations are local over TM. We have chosen a closed cone domain because the causality aspects of these theories can be studied with the help of the theory of differential inclusions.

This paper is concerned with a special type of pseudo-Finsler space, namely those for which all pseudo-Minkowskian tangent spaces $\left(T_{x} M, \mathscr{L}_{x}\right)$ are modeled on the same pseudo-Minkowski space $(V, L)$. This means that locally we can find a linear isomorphism (sufficiently differentiable in $x \in U \subset M$ )

$$
\varphi_{x}: T_{x} M \rightarrow V,
$$

such that $\varphi_{x}\left(\Omega_{x}\right)=\omega$, and $L\left(\varphi_{x}(y)\right)=\mathscr{L}_{x}(y)$ for every $y \in \bar{\Omega}_{x}$. Parallelizable manifolds admit structures of this type and parallelizability can be relaxed provided $(V, L)$ admits a Lie group $G$ of linear isomorphisms (they preserve $\mathscr{L}$ and its domain). In that case it is sufficient that $M$ admits a $G$-structure [3. Our considerations will be of local character so we shall not enter into a more detailed discussion [3]. The idea of Finsler space modeled on the same Minkowski space has been first introduced and investigated by Ichijyo [3] and further progress has been obtained by Aikou in [4. Matsumoto [5], Izumi [6], Sakaguchi [7, and Asanov and Kirnasov [8] had also considered similar spaces referring to them 
as 1-form Finsler spaces. Szilasi and Tamássy call them affine deformations of Minkowski spaces 9, while Libing Huang, and Bartelmeß and Matveev borrow from Zhongmin Shen the terminology single colored or monochromatic Finsler spaces [10, 11].

Roughly speaking, while in pseudo-Finsler spaces the anisotropic features change from point to point and can even be absent in some open subset of $M$, in pseudo-Finsler spaces modeled on a pseudo-Minkowski space the anisotropic features do not depend on the point. Of course, pseudo-Riemannian manifolds provide an example of pseudo-Finsler space modeled on a pseudo-Minkowski space. Berwald spaces are also modeled on the same Minkowski space. The linear isometry between different tangent spaces is provided by the Ehresmann non-linear (actually linear) parallel transport connecting the two points. For Berwald spaces the Ehresmann connection is nothing but the pullback of the Berwald connection under any local section.

However, Berwald spaces are quite rigid. In fact, let $G_{x}$ be the group of linear isomorphisms of $\left(T_{x} M, \mathscr{L}_{x}\right)$ and let $\mathfrak{g}_{x}$ be its Lie algebra. Let $R_{x}: \Lambda^{2} T_{x} M \rightarrow$ $\operatorname{End}\left(T_{x} M\right)$ be the curvature of the Ehresmann connection. We have (for related ideas cf. [12])

Theorem 1. Let $(M, \mathscr{L})$ be a connected Berwald pseudo-Finsler space. Then the Lie algebra generated by $R_{x}$ is contained in $\mathfrak{g}_{x}$. Moreover, if for every $x \in M, \mathfrak{g}_{x}=0$ (e.g. because it is modeled on the same pseudo-Minkowski space with no symmetries), then the Berwald pseudo-Finsler space is locally an open subset of pseudo-Minkowski space, while if additionally $G_{x}$ is just the identity for every $x$, then it is globally an open subset of pseudo-Minkowski space.

Proof. Since the space is Berwald the transport with the Ehresmann connection is a linear isomorphism of tangent pseudo-Minkowski spaces (it preserves also the Finsler Lagrangian [13, Prop. 10.1.1][14, Prop. 2.1]). Let $X, Y \in T_{x} M$. By the Ambrose-Singer theorem $R_{x}(X, Y)$ is an element of the Lie Algebra of $\operatorname{Hol}_{x}$, the holonomy Lie group of the connection at $x$. Thus $t \mapsto \exp \left[t R_{x}(X, Y)\right]$ is a one parameter group of linear isomorphisms of $\left(T_{x} M, \mathscr{L}_{x}\right)$, hence $R_{x}(X, Y) \subset \mathfrak{g}_{x}$.

If for every $x, \mathfrak{g}_{x}=0$ then $R=0$ and since the Ehresmann connection is torsionless it follows that the space is locally pseudo-Minkowski (i.e. $M$ is covered by patches $U_{i}$ such that over $T U_{i}$ in the induced tangent coordinates $\mathscr{L}$ has no dependence on $x$ ), see e.g. [15], hence modeled on the same pseudoMinkowski space. If for every $x, G_{x}$ is just the identity it is an open subset of pseudo-Minkowski space since the transition map in the intersection of two patches must belong to $G$, the linear group of isomorphisms of the model pseudoMinkowski space, which is trivial.

This result shows that Berwald spaces can be non-trivial only if the model pseudo-Minkowski space has a non-trivial linear group of symmetries, a fact which limits considerably the family of these spaces. The theorem is quite useful, for instance it immediately implies that a 2-dimensional Berwald Randers space is locally a Riemannian space or an open subset of a Minkowski space, with no need for calculations, in fact the indicatrix is a translated circle and there is 
no non-trivial linear Lie group of symmetries which send the indicatrix to itself unless there is no translation of the center (the same result could have been obtained using [16, Th. 2.4.1 (ii)]).

In recent years there has been a growing interest in Finsler geometry. Unfortunately, in applications the calculations are often prohibitive so it is natural to look for a smaller family of spaces for which they become amenable.

In this work we shall be interested in pseudo-Finsler spaces modeled on a Minkowski space which are not necessarily Berwald. We prove that most tensors of interest can be explicitly calculated. Namely, we shall show that they can be reduced to a form factored into terms depending on the base coordinates $x^{\mu}$ or on the vertical coordinates $y^{a}$ of the model pseudo-Mikowski space. This very fact seems to have passed unnoticed, though it implies a dramatic simplification in the investigation of these spaces. Also it must be remarked that this family, though smaller than the general family of pseudo-Finsler spaces, is in the end fairly large. Most proposed metrics in applications are of this form, we mention the Berwald-Moór metric [17, 18, Bogoslovsky and Goenner's metric [19, 20], Bogoslovsky's metric [21, 22], the conic metric introduced by the second author in 23], the Randers spaces in which the $\beta 1$-form has constant $\alpha$-module, or the pp-wave Finsler metric by Fuster and Pabst [24] which we shall more closely study in the last section.

One Finslerian quantity which attracted our interest is the Ricci scalar. In the theory of Finsler gravity most proposals for the vacuum dynamical equations, starting from Horvath's [25], imply the condition

$$
\operatorname{Ric}(y)=0 \text {, }
$$

which is also suggested by studies of the Raychaudhuri equation [26, 14]. Although some of the final tensorial expressions which we shall present are rather concise, occasionally we had to pass through computations involving hundreds of terms. This happened precisely in the calculation of $\operatorname{Ric}(y)$ so we are happy to have been able to reduce its expression to a much simpler form, cf. Eq. (16). Here we have been helped by the $x$ Act suite of tensor computer algebra [27].

\section{The strategy}

Let $\left\{\tilde{e}_{a}\right\}$ be a basis of $V$ and let $y^{a}$ be the induced coordinates, so if $\tilde{y} \in V$, then there are $y^{a} \in \mathbb{R}, a=1, \ldots, m$, such that $\tilde{y}=y^{a} \tilde{e}_{a}$ (we adopt the Einstein summation convention). Let us define at $x \in M$,

$$
y:=\varphi_{x}^{-1}(\tilde{y}), \quad e_{a}:=\varphi_{x}^{-1}\left(\tilde{e}_{a}\right),
$$

so that $y=y^{a} e_{a}$.

Let us denote with $\partial_{\mu}$ the basis of $T_{x} M$ induced by a local coordinate system $\left\{x^{\mu}\right\}$ on $U \subset M$, then there are invertible matrices (vierbein) $e_{a}^{\mu}$ and $e_{\mu}^{a}$, such that $e_{a}=e_{a}^{\mu} \partial_{\mu}, e^{a}=e_{\mu}^{a} \mathrm{~d} x^{\mu}$, where $\left\{e^{a}\right\}$ is the dual basis to $\left\{e_{a}\right\}$,

$$
e_{\mu}^{a} e_{b}^{\mu}=\delta_{b}^{a}, \quad e_{a}^{\mu} e_{\nu}^{a}=\delta_{\nu}^{\mu}
$$


The vector $y \in T_{x} M$ can be expanded as follows

$$
y=y^{\mu} \partial_{\mu}=y^{a} e_{a},
$$

where (here we use the physicist convention according to which a different type of index, say Greek, Roman, might distinguish different objects)

$$
y^{\mu}=y^{a} e_{a}^{\mu} .
$$

We shall call $y^{a}$ the vertically induced internal variables. The commutation coefficients $\left[e_{a}, e_{b}\right]=c^{c}{ }_{a b} e_{c}$ are

$$
c_{a b}^{c}=\left(e_{a}^{\alpha} e_{b, \alpha}^{\mu}-e_{b}^{\alpha} e_{a, \alpha}^{\mu}\right) \partial_{\mu}=e_{a}^{\alpha} e_{b}^{\beta}\left(e_{\alpha, \beta}^{c}-e_{\beta, \alpha}^{c}\right) .
$$

where in the second equality we used (2). They can also be obtained through the identity

$$
\mathrm{d} e^{c}=\mathrm{d}\left(e_{\gamma}^{c} \mathrm{~d} x^{\gamma}\right)=-c_{a b}^{c} \frac{1}{2} e^{a} \wedge e^{b} .
$$

We shall also denote $c_{a b, d}^{c}=c_{a b, \mu}^{c} e_{d}^{\mu}$.

Now according to the isomorphism assumption between $\left(T_{x} M, \mathscr{L}_{x}\right)$ and $(V, L)$ we have

$$
\mathscr{L}_{x}\left(y^{a} e_{a}\right)=L\left(y^{a} \tilde{e}_{a}\right) .
$$

In what follows $\tilde{e}_{a}$ is considered chosen and fixed once and for all, so we can regard $L$ as a function $L: \mathbb{R}^{m} \rightarrow \mathbb{R}$. So we denote

$y_{a}=\partial_{a} L, \quad g_{a b}=\partial_{a} \partial_{b} L, \quad C_{a b c}=\frac{1}{2} \partial_{a} \partial_{b} \partial_{c} L, \quad I_{a}=g^{b c} C_{a b c}, \quad C_{a b c d}=\partial_{a} C_{b c d}$,

called respectively, the Legendre dual of $y$, the (pseudo-)Finsler metric, the Cartan torsion, the mean Cartan torsion and the Cartan curvature of the tangent pseudo-Minkowski space. Since the metric is non-degenerate we shall also have the inverse metric $g^{a b}$. To simplify notations we shall also regard $\mathscr{L}$ as a local function in the horizontally induced variables i.e. from $U \times \mathbb{R}^{m} \rightarrow \mathbb{R}, U \subset \mathbb{R}^{m}$, so that $\left(x^{\mu}, y^{\nu}\right) \rightarrow \mathscr{L}\left(x^{\mu}, y^{\nu}\right)$. Thus we can write

$$
\mathscr{L}\left(x^{\mu}, y^{\nu}\right)=L\left(e_{\nu}^{a}(x) y^{\nu}\right) .
$$

Now, most references of Finsler geometry provide expressions of the most important tensors in the coordinates $\left(x^{\mu}, y^{\nu}\right)$ induced over the tangent bundle by the coordinates on the base, so we shall stick to those [15, 28, 29, 30]. However, our aim is to change variables from the pair $\left(x^{\mu}, y^{\nu}\right)$ to $\left(x^{\mu}, y^{a}\right)$. So we shall need the change of partial derivatives

$$
\begin{aligned}
& \left.\left.\frac{\partial}{\partial y^{\alpha}}\right)_{x^{\gamma}}=e_{\alpha}^{a} \frac{\partial}{\partial y^{a}}\right)_{x^{\gamma}}, \\
& \left.\left.\left.\frac{\partial}{\partial x^{\alpha}}\right)_{y^{\gamma}}=\frac{\partial}{\partial x^{\alpha}}\right)_{y^{c}}+e_{\beta, \alpha}^{b} e_{c}^{\beta} y^{c} \frac{\partial}{\partial y^{b}}\right)_{x^{\gamma}} .
\end{aligned}
$$


The idea is to arrange the notable tensors in terms of the quantities $e_{\alpha}^{a}, e_{a}^{\alpha}$, $c_{b c}^{a}, c_{b c, d}^{a}$ which only depend on $x^{\mu}$ and in terms of the quantities displayed in (4) including the inverse metric $g^{a b}$ which only depend on $y^{a}$. So we do not raise or lower indices with the metric in the commutation coefficients otherwise we would spoil this property introducing objects dependent on both variables. Only after we have obtained all expressions of interest we simplify them lowering or raising the indices of the commutators, with the convention that the upper index in $c_{b c}^{a}$ gets lowered to the left as $c_{a b c}$. For instance applying the previous formulas to $\mathscr{L}$ we get

$$
\begin{aligned}
y_{\alpha}:= & \frac{\partial \mathscr{L}}{\partial y^{\alpha}}=y_{a} e_{\alpha}^{a}, \\
& \frac{\partial \mathscr{L}}{\partial x^{\alpha}}=y_{b} e_{\beta, \alpha}^{b} e_{c}^{\beta} y^{c} .
\end{aligned}
$$

These derivatives enter the calculation of the spray [29, Eq. (5.2)]

$$
\begin{aligned}
G^{\mu} & :=\frac{1}{2} g^{\mu \sigma}\left(\frac{\partial^{2} \mathscr{L}}{\partial x^{\rho} \partial y^{\sigma}} y^{\rho}-\frac{\partial \mathscr{L}}{\partial x^{\sigma}}\right) \\
& =e_{m}^{\mu}\left[\frac{1}{2}\left(g^{m k} y_{q} y^{n} c^{q}{ }_{k n}+e_{\delta, \rho}^{m} e_{d}^{\delta} e_{p}^{\rho} y^{d} y^{p}\right)\right] .
\end{aligned}
$$

Observe that all terms either depend on $x^{\mu}$ or $y^{a}$; this fact makes the next calculations easier by repeatedly using (5)-(6).

The coefficients of the non-linear connection are

$$
\begin{aligned}
N_{\alpha}^{\mu}:=\frac{\partial G^{\mu}}{\partial y^{\alpha}}=e_{l}^{\mu} e_{\alpha}^{b}\left[e_{\rho, \eta}^{l} e_{b}^{\eta} e_{p}^{\rho} y^{p}+c_{k n}^{m}\left(-C_{b}^{l k} y_{m} y^{n}\right.\right. \\
\\
\left.\left.+\frac{1}{2}\left(g^{l k} g_{m b} y^{n}+g^{l k} y_{m} \delta_{b}^{n}+\delta_{m}^{l} \delta_{b}^{k} y^{n}\right)\right)\right] .
\end{aligned}
$$

The coefficients of the Berwald Finsler connection are

$$
\begin{aligned}
2 G_{\alpha \beta}^{\rho}:= & 2 \frac{\partial^{2} G^{\rho}}{\partial y^{\alpha} \partial y^{\beta}}=\left[e_{b}^{\rho}\left(e_{\alpha, \beta}^{b}+e_{\beta, \alpha}^{b}\right)\right] \\
& +c^{e}{ }_{m n}\left(g^{r m} g_{e a} \delta_{b}^{n}+g^{r m} g_{e b} \delta_{a}^{n}+2 g^{r m} C_{e a b} y^{n}-2 C_{b}^{r m} g_{e a} y^{n}\right. \\
& \left.-2 C_{a}^{r m} g_{e b} y^{n}-2 C_{a}^{r m} y_{e} \delta_{b}^{n}-2 C_{b}^{r m} y_{e} \delta_{a}^{n}-2\left(\frac{\partial}{\partial y^{b}} C_{a}^{r m}\right) y_{e} y^{n}\right) .
\end{aligned}
$$

We could simplify the vertical derivative with

$$
-\frac{1}{2} \frac{\partial}{\partial y^{b}} \frac{\partial}{\partial y^{a}} g^{r m}=\frac{\partial}{\partial y^{b}} C_{a}^{r m}=C_{a b}^{r m}-2 C_{b}^{r s} C_{s a}^{m}-2 C_{b}^{m s} C_{s a}^{r},
$$

but the expression gets longer. The Berwald curvature tensor is defined through $G_{\alpha \beta \gamma}^{\rho}=\frac{\partial^{3} G^{\rho}}{\partial y^{\alpha} \partial y^{\beta} \partial y^{\gamma}}$ and in our case it is given by

$$
2 G_{a b c}^{r}=2 G_{\alpha \beta \gamma}^{\rho} e_{\rho}^{r} e_{a}^{\alpha} e_{b}^{\beta} e_{c}^{\gamma}=c_{m n}^{e} \frac{\partial^{3}}{\partial y^{a} \partial y^{b} \partial y^{c}}\left(g^{r m} y_{e} y^{n}\right) .
$$


Here it is not convenient to expand the derivatives. The mean Berwald curvature $E_{b c}:=\frac{1}{2} G_{r b c}^{r}$ follows from

$$
G_{r b c}^{r}=-c_{m n}^{e} \frac{\partial^{2}}{\partial y^{b} \partial y^{c}}\left(I^{m} y_{e} y^{n}\right) .
$$

The Douglas curvature is defined by $D_{\alpha \beta \gamma}^{\rho}=\frac{\partial^{3}}{\partial y^{\alpha} \partial y^{\beta} \partial y^{\gamma}}\left(G^{\rho}-\frac{1}{m+1} N_{a}^{a} y^{\rho}\right)$ and in our case it is given by

$$
D_{a b c}^{r}=c_{p q}^{e} \frac{\partial^{3}}{\partial y^{a} \partial y^{b} \partial y^{c}}\left(\left(\frac{1}{2} g^{r p}+\frac{1}{m+1} I^{p} y^{r}\right) y_{e} y^{q}\right)
$$

where $m$ is the dimension of the Finsler space. In the next expression $\nabla^{V C}$ is the vertical Cartan derivative (whose connection coefficients in coordinates $\left\{y^{a}\right\}$ are $\left.C_{a b}^{c}\right)$ and $h_{b}^{a}:=\delta_{b}^{a}-\frac{1}{g_{y}(y, y)} y^{a} y_{b}, g_{y}(y, y):=2 L$ is the usual projection on the space tangent to the indicatrix.

The Landsberg tensor is

$$
\begin{aligned}
L_{a b c}= & L_{\alpha \beta \gamma} e_{a}^{\alpha} e_{b}^{\beta} e_{c}^{\gamma}=-\frac{1}{2} y_{r} G_{a b c}^{r}=-\frac{1}{4} c_{m n}^{e} y_{r} \frac{\partial^{3}}{\partial y^{a} \partial y^{b} \partial y^{c}}\left(g^{r m} y_{e} y^{n}\right) \\
= & -\frac{1}{2} c^{e}{ }_{m n}\left(y^{m} C_{e b c} \delta_{a}^{n}+y^{m} C_{e c a} \delta_{b}^{n}+y^{m} C_{e a b} \delta_{c}^{n}\right. \\
& +C_{b c}^{m} g_{e a} y^{n}+C_{c a}^{m} g_{e b} y^{n}+C_{a b}^{m} g_{e c} y^{n} \\
& +C_{b c}^{m} y_{e} \delta_{a}^{n}+C_{c a}^{m} y_{e} \delta_{b}^{n}+C_{a b}^{m} y_{e} \delta_{c}^{n} \\
& \left.+2\left(C_{a b c}^{m}-C_{s a}^{m} C_{b c}^{s}-C_{s c}^{m} C_{a b}^{s}-C_{s b}^{m} C_{c a}^{s}\right) y_{e} y^{n}\right) \\
= & k_{m n e} y^{e}\left(C_{b c}^{m} \delta_{a}^{n}+C_{c a}^{m} \delta_{b}^{n}+C_{a b}^{m} \delta_{c}^{n}\right) \\
& +k_{m n e} y^{n} y^{e}\left(C_{a b c}^{m}-C_{b c}^{s} C_{a s}^{m}-C_{c a}^{s} C_{b s}^{m}-C_{a b}^{s} C_{c s}^{m}\right) \\
= & k_{m n e} y^{e}\left[C_{b c}^{[m} h_{a}^{n]}+C_{c a}^{[m} h_{b}^{n]}+C_{a b}^{[m} h_{c}^{n]}+y^{[n} h^{m] s} h_{a}^{p} h_{b}^{q} h_{c}^{r} \nabla_{s}^{V C} C_{p q r}\right] .
\end{aligned}
$$

The first expressions are particularly suited for calculations since the whole $x$ dependence is contained in the first $c_{m n}^{e}$ factor, while the subsequent factors depend only on $y^{a}$. In the last two expressions we introduced the object antisymmetric in the first two indices (they are a sort of Ricci rotation coefficients)

$$
k_{m n e}(x, y)=\frac{1}{2}\left(c_{m n e}+c_{n e m}-c_{e m n}\right) .
$$

The last expression in Eq. (14) is useful because through contraction with $y_{n}$ it shows that for $m \geq 3$ the tensor in square bracket vanishes if and only if $C_{a b c}=0$. Thus it is not possible to find vertical conditions weaker than $C_{a b c}=0$ (i.e. the pseudo-Riemannian case) which guarantee that $L_{a b c}=0$.

Still the expression for $L$ simplifies considerably when $h_{a}^{p} h_{b}^{q} h_{c}^{r} h_{d}^{s} \nabla_{s}^{V C} C_{p q r}=$ 0 which happens if and only if the geometry of the indicatrix is such that $\nabla^{\mathfrak{h}} \mathfrak{c}=0$ where $\mathfrak{c}$ is the Pick cubic form and $\mathfrak{h}$ is the affine metric for the centroaffine transverse (for translation between Finsler and centroaffine geometry the reader is referred to 31]). For instance, $\nabla^{\mathfrak{h}} \mathfrak{c}=0$ holds true whenever the indicatrix is a homogeneous affine sphere [32, 33. 
The mean Landsberg curvature is

$$
\begin{aligned}
J_{c}:=L_{a c}^{a} & =-\frac{1}{2} c_{m n}^{e}\left(y^{m} I_{e} \delta_{c}^{n}+I^{m} g_{e c} y^{n}+I^{m} y_{e} \delta_{c}^{n}+2\left(\frac{\partial}{\partial y^{c}} I^{m}+C_{s c}^{m} I^{s}\right) y_{e} y^{n}\right) \\
& =k_{m n e} y^{e}\left[I^{m} \delta_{c}^{n}+y^{n}\left(\frac{\partial}{\partial y^{c}} I^{m}+C_{s c}^{m} I^{s}\right)\right] \\
& =k_{m n e} y^{e}\left[h_{c}^{[n} I^{m]}+y^{[n}\left(h_{c}^{a} h_{b}^{m]} \nabla_{a}^{V C} I^{b}\right)\right] .
\end{aligned}
$$

Again, the first expression is useful in calculations since it factors the $x^{\mu}$ and $y^{a}$ dependence.

The last formula can be used to show that the vertical tensor in square brackets vanishes if and only if $I=0$ (for the 'only if' direction contract first with $y_{n}$ ). Thus it is not possible to find vertical conditions weaker than $I_{c}=0$ which guarantee that $J_{c}=0$ (a fact known to hold in general for any pseudoFinsler space).

The curvature of the non-linear connection is

$$
R_{\alpha \beta}^{\mu}:=\frac{\delta N_{\beta}^{\mu}}{\delta x^{\alpha}}-\frac{\delta N_{\alpha}^{\mu}}{\delta x^{\beta}}, \quad \frac{\delta}{\delta x^{\alpha}}:=\frac{\partial}{\partial x^{\alpha}}-N_{\alpha}^{\mu} \frac{\partial}{\partial y^{\mu}} .
$$

Our calculation for the Ricci scalar gives

$$
\begin{aligned}
\operatorname{Ric}(y):= & R_{\mu \alpha}^{\mu} y^{\alpha}=2 \frac{\partial G^{\mu}}{\partial x^{\mu}}-y^{\mu} \frac{\partial^{2} G^{\nu}}{\partial x^{\mu} \partial y^{\nu}}+2 G^{\mu} \frac{\partial^{2} G^{\nu}}{\partial y^{\mu} \partial y^{\nu}}-\frac{\partial G^{\mu}}{\partial y^{\nu}} \frac{\partial G^{\nu}}{\partial y^{\mu}} \\
= & g^{a d} y^{b} y_{c} c^{c}{ }_{a b, d}+y^{a} y^{b} c^{c}{ }_{b c, a}+I^{b} y^{a} y^{c} y_{d} c^{d}{ }_{b c, a} \\
& +c^{i}{ }_{j k} c^{l}{ }_{m n} g_{i a} g_{l b}\left(\frac{1}{4} g^{j m} g^{k n} y^{a} y^{b}+g^{b n} g^{k m} y^{a} y^{j}-\frac{1}{2} g^{a n} g^{b k} y^{j} y^{m}\right. \\
& -\frac{1}{2} g^{a b} g^{k n} y^{j} y^{m}+2 y^{a} y^{j} y^{m} C^{b k n}-y^{a} y^{j} y^{b} y^{m} C_{h}^{k g} C_{g}^{n h} \\
& \left.+I^{j} g^{k n} y^{a} y^{b} y^{m}-g^{b k} I^{n} y^{a} y^{j} y^{m}-g^{e n} y^{a} y^{j} y^{b} y^{m} \frac{\partial}{\partial y^{e}} I^{k}\right) .
\end{aligned}
$$

It can be easily checked that if the metric does not depend on the vertical variable $\left(C_{a b c}=0\right)$ we get the usual expression of the Ricci tensor in terms of the commutation coefficients [34, Sect. 98].

\section{The Bogoslovsky model Lorentz-Minkowski space}

A Lorentz-Minkowski space that has attracted considerable interest is Bogoslovsky's 22] namely $L=-\frac{1}{2} F^{2}$, where

$$
F=\left(2 y^{0} y^{n}-\sum_{J=1}^{n-1}\left(y^{J}\right)^{2}\right)^{(1-b) / 2}\left(y^{0}\right)^{b}
$$

and $b$ is a dimensionless parameter. Its study for $n=3$ was revived by the Very Special Relativity theory by Cohen and Glashow 35, who observed that most observations are really compatible with a subgroup $S I M(2)$ of the Lorentz 
group, and by Gibbons, Gomis and Pope [36] who showed that among the possible continuous deformations $D I S I M_{b}(2)$ is particularly interesting and leads to the local Lagrangian of a Lorentz-Finsler geometry based on the Bogoslovsky Lorentz-Minkowski space.

In this section we apply our method to the Bogoslovsky metric, and in a next section we specialize to a recent pp-wave Lorentz-Finsler space based on it. It will be convenient to set

$$
A=2 y^{0} y^{n}-\sum_{J=1}^{n-1}\left(y^{J}\right)^{2}=2 y^{0} y^{n}-\delta_{J K} y^{J} y^{K},
$$

In what is to follow capital Latin indices $J, K, L$ run from 1 to $n-1$ and $\delta_{J K}$ (resp. $\delta^{J K}$ ) represents the Euclidean metric (resp. its inverse) in dimension $n-1$. We proceed now with the vertical derivatives. The differential of $L=-F^{2} / 2$ is

$$
\begin{aligned}
& y_{0}=\frac{F^{2}}{A y^{0}}\left(-b A+(b-1) y^{0} y^{n}\right), \\
& y_{J}=\frac{F^{2}}{A}(1-b) y^{J}, \\
& y_{n}=-\frac{F^{2}}{A}(1-b) y^{0} .
\end{aligned}
$$

The components of the metric are

$$
\begin{aligned}
g_{00} & =b\left(\frac{F}{A y^{0}}\right)^{2}\left[2(1-b)\left(y^{0} y^{n}\right)^{2}+4(b-1) y^{0} y^{n} A+(1-2 b) A^{2}\right], \\
g_{0 J} & =2(b-1) b \frac{F^{2}}{A^{2}} \frac{y^{J}}{y^{0}}\left(y^{0} y^{n}-A\right), \\
g_{0 n} & =(1-b) \frac{F^{2}}{A^{2}}\left(-(1+2 b) A+2 b y^{0} y^{n}\right), \\
g_{J K} & =(1-b) \frac{F^{2}}{A^{2}}\left(2 b y^{J} y^{K}+A \delta_{J K}\right), \\
g_{J n} & =2(b-1) b \frac{F^{2}}{A^{2}} y^{0} y^{J}, \\
g_{n n} & =2(1-b) b \frac{F^{2}}{A^{2}}\left(y^{0}\right)^{2} .
\end{aligned}
$$

The components of its inverse are

$$
\begin{aligned}
g^{00} & =-\frac{2 b\left(y^{0}\right)^{2}}{(1+b) F^{2}}, \\
g^{0 J} & =-\frac{2 b y^{0} y^{J}}{(1+b) F^{2}}, \\
g^{0 n} & =-\frac{A}{(1+b) F^{2}}-\frac{2 b y^{0} y^{n}}{(1+b) F^{2}}, \\
g^{J K} & =\frac{A}{(1-b) F^{2}} \delta^{J K}-\frac{2 b y^{J} y^{K}}{(1+b) F^{2}}, \\
g^{J n} & =\frac{2 b y^{J} A}{\left(1-b^{2}\right) F^{2} y^{0}}-\frac{2 b y^{J} y^{n}}{(1+b) F^{2}}, \\
g^{n n} & =\frac{b A\left(A-4 y^{0} y^{n}\right)}{\left(b^{2}-1\right) F^{2}\left(y^{0}\right)^{2}}-\frac{2 b\left(y^{n}\right)^{2}}{(1+b) F^{2}} .
\end{aligned}
$$

Remark 2. The components of the Cartan torsion are too many to be displayed here. Here we just give the mean Cartan torsion for $n=3$

$$
I_{0}=4 b\left(y^{0}\right)^{-1}, \quad I_{1}=4 b y^{1} / A, \quad I_{2}=4 b y^{2} / A, \quad I_{3}=-4 b y^{0} / A,
$$


its square and that of the Cartan torsion

$$
I_{a} I^{a}=-\frac{16 b^{2} A^{b-1}}{\left(b^{2}-1\right)\left(y^{0}\right)^{2 b}}, \quad C_{a b c} C^{a b c}=-\frac{10 b^{2} A^{b-1}}{\left(b^{2}-1\right)\left(y^{0}\right)^{2 b}} .
$$

Observe that according to our method, we have obtained some characteristic tensors of the model pseudo-Minkowski space, only dependent on $y^{a}$, and so we have just to calculate the commutation coefficients of the vierbein for those Lorentz-Finsler spaces on which we are interested, we shall see examples in theorem 2 and in Sec. 4.

\subsection{The Berwald condition}

In this section the dimension $n+1$ is arbitrary. Due to Eq. (77) a pseudo-Finsler space is Berwald iff

$$
H^{r}:=\frac{1}{2} c_{b c}^{a}(x) y_{a}\left(g^{r b} y^{c}-g^{r c} y^{b}\right)
$$

is quadratic in $y^{a}$. Given a triple $(a ; b, c)$ let us say that it has property $*$ if

$$
H_{a}^{r b c}:=y_{a}\left(g^{r b} y^{c}-g^{r c} y^{b}\right)
$$

is quadratic in $y^{d}$ for every $r$. In order to prove that a space is Berwald it is sufficient to show that $c_{b c}^{a}(x) \neq 0$ only for those triples $(a ; b, c)$ for which $*$ holds true.

Remarkably, for the Bogoslovsky Lorentz-Minkowski space $*$ is true for every choice $(a ; b, c)$ with $a>0$ and $b, c \neq n$, namely

$$
y_{a}\left(y^{0} g^{r J}-y^{J} g^{r 0}\right), \quad y_{a}\left(y^{J} g^{r K}-y^{K} g^{r J}\right)
$$

are quadratic for every $r$. This fact can be checked with a calculation but can be also understood as follows. Notice from (19) that $y_{a}$ for $a>0$ is proportional to $l_{a}\left(y^{d}\right) F^{2} / A$ where $A$ is given by (18) and $l_{a}\left(y^{d}\right)$ is some linear function. The inverse metric (21) includes a last term that cancels out when the metric is inserted into $H_{a}^{r b c}$, so only the other term proportional to $A / F^{2}$ does not cancel. The factors simplify and only a quadratic term is left.

Less obvious is the fact that $*$ is true for $(n ; n, 0)$ (or equivalently $(n ; 0, n)$ ), but it can be easily checked with a calculation.

$$
\begin{aligned}
& H_{n}{ }^{0 n 0}=\frac{(1-b)}{1+b}\left(y^{0}\right)^{2}, \quad H_{n}{ }^{J n 0}=-\frac{2 b}{1+b} y^{0} y^{J}, \\
& H_{n}{ }^{n n 0}=\frac{b A-(1+3 b) y^{0} y^{n}}{1+b} .
\end{aligned}
$$

As a consequence, our method allows us to prove the following theorem

Theorem 2. Any $n+1$-dimensional Lorentz-Finsler space of coordinates $\left\{u, v,\left\{x^{i}\right\}_{i=1, \cdots n-1}\right\}$ and modeled on the Bogoslovsky Lorentz-Minkowski space

$$
F=\left[2 y^{0} y^{n}-\Sigma_{i=1}^{n-1}\left(y^{i}\right)^{2}\right]^{(1-b) / 2}\left(y^{0}\right)^{b},
$$


for which the vierbein can be chosen in the local form

$$
\begin{aligned}
y^{0} & =U(u) \mathrm{d} u, \\
y^{i} & =\sum_{j=1}^{n-1} Z_{j}^{i}(\vec{x}, u) \mathrm{d} x^{j}+Z^{i}(\vec{x}, u) \mathrm{d} u, \quad i=1, \ldots, n-1 \\
y^{n} & =\mathrm{d} v+V(\vec{x}, u, v) \mathrm{d} u+\sum_{j=1}^{n-1} V_{j}(\vec{x}, u) \mathrm{d} x^{j} .
\end{aligned}
$$

(where $U, Z_{j}^{i}, Z^{i}, V, V_{j}$ are arbitrary $C^{1}$ functions, with $Z_{j}^{i}$ invertible and $U \neq 0$ ) is really Berwald.

Proof. Using these vierbein an explicit computation shows that

$$
\begin{aligned}
& d y^{0}=0 \\
& d y^{J}=-\frac{1}{2} c_{K L}^{J}(x) y^{K} \wedge y^{L}-c_{K 0}^{J}(x) y^{K} \wedge y^{0}, \\
& d y^{n}=-c_{J 0}^{n}(x) y^{J} \wedge y^{0}-\frac{1}{2} c_{J K}^{n}(x) y^{J} \wedge y^{K}-c_{n 0}^{n}(x) y^{n} \wedge y^{0} .
\end{aligned}
$$

Therefore, we deduce that the property $*$ holds for the triples $(a ; b, c)$ corresponding to the only non-vanishing commutation coefficients $c_{b c}^{a}$.

\section{An example: Finsler pp-wave}

We end the paper with an application of the previous theory. Recently Fuster and Pabst have proposed a Finslerian gravitational pp-wave 24] based on the Bogoslowsky model Lorentz-Minkowski space. Their vierbein are

$$
y^{0}=\mathrm{d} u, \quad y^{1}=\mathrm{d} x, \quad y^{2}=\mathrm{d} y, \quad y^{3}=\mathrm{d} v+\frac{1}{2} \Phi(x, y, u) \mathrm{d} u .
$$

Here $\{u, x, y, v\}$ are coordinates on $M$, which would be denoted $\left\{x^{\alpha}\right\}$ in the notation of the previous sections, the differentials $\{\mathrm{d} u, \mathrm{~d} x, \mathrm{~d} y, \mathrm{~d} v\}$ are nothing but the coordinates of the tangent bundle denoted by $\left\{y^{\alpha}\right\}$ in the previous sections, while the previous line in display gives $\left\{y^{a}\right\}$, namely the vertically induced coordinates which are used to clarify that the Finsler function is indeed based on the same Minkowski space. In fact Eq. (17), which has no dependence on the base variable $x$, holds true by definition. The only non-vanishing commutation coefficients are obtained with the exterior differentiation of $y^{3}$, see Eq. (3) namely

$$
c_{01}^{3}=-c_{10}^{3}=\Phi_{x}, \quad c_{02}^{3}=-c_{20}^{3}=\Phi_{y} .
$$

At this point we have just to insert these quantities into the expressions of the notable tensors.

By our Theorem 2 the Lorentz-Finsler space is Berwald. In fact, for the ppwave spacetime the components of the spray computed according to equation 
(77) yield

$$
\begin{aligned}
G^{u} & =0 \\
G^{x} & =\frac{1}{4}\left(y^{0}\right)^{2} \Phi_{x}, \\
G^{y} & =\frac{1}{4}\left(y^{0}\right)^{2} \Phi_{y}, \\
G^{v} & =\frac{1}{4} y^{0}\left(y^{0} \Phi_{u}+2 y^{2} \Phi_{y}+2 y^{1} \Phi_{x}\right),
\end{aligned}
$$

which are quadratic. Since the space is Berwald, the Landsberg tensor and the Douglas curvature vanish: $L_{a b c}=0, D_{b c d}^{a}=0$.

The Ricci scalar computed according to (16) is given by

$$
\operatorname{Ric}(y)=\frac{1}{2}\left(y^{0}\right)^{2}\left(\Phi_{y y}+\Phi_{x x}\right) .
$$

So it vanishes iff $\Phi$ is harmonic.

\section{Conclusions}

We have shown that for pseudo-Finsler spaces modeled on a pseudo-Minkowski space several Finslerian quantities can be explicit calculated. We have found interesting expressions for the Berwald curvature, Douglas curvature, Landsberg tensor, mean Landsberg tensor, mean Berwald curvature, and Ricci scalar in terms of: (a) vertical quantities, which only depend on the geometry of the indicatrix, and (b) base quantities, namely the commutation coefficients, which only depend on how the model Minkowski space is displaced all over the manifold. These different contributions can be separately investigated for specific metrics. As an illustration, we have applied the method to the Finslerian ppwave recently found by Fuster and Pabst. We hope that our results could serve to bridge the gap between abstract Finsler geometry and its applications.

\section{Acknowledgements}

AGP wishes to thank the "Dipartimento di Matematica e Informatica U. Dini" at Florence University where this work was carried out for hospitality and financial support.

AGP is supported by the projects IT956-16 ("Eusko Jaurlaritza", Spain), FIS2014-57956-P ("Ministerio de Economía y Competitividad", Spain) and PTDC/MAT-ANA /1275/2014 ("Fundação para a Ciência e a Tecnologia", Portugal). EM wishes to thank GNFM of INDAM.

\section{References}

[1] H. Martini, K. J. Swanepoel, and G. Weiß, The geometry of Minkowski spaces-a survey. I, Expo. Math. 19, 97 (2001). 
[2] J. C. Álvarez Paiva and A. C. Thompson, Volumes on normed and Finsler spaces, in A sampler of Riemann-Finsler geometry, Vol. 50 of Math. Sci. Res. Inst. Publ. (Cambridge Univ. Press, Cambridge, 2004), p. 1.

[3] Y. Ichijyō, Finsler manifolds modeled on a Minkowski space, J. Math. Kyoto Univ. 16, 639 (1976).

[4] T. Aikou, Some remarks on Finsler vector bundles, Publ. Math. Debrecen $\mathbf{5 7}, 367$ (2000).

[5] M. Matsumoto, On Finsler spaces with 1-form metric, Tensor N.S. 32, 161 (1978).

[6] H. Izumi, Nonholonomic frames in a Finsler space with a 1-form metric, Tensor (N.S.) 40, 189 (1983).

[7] T. Sakaguchi, Remarks on Finsler spaces with 1-form metric, Tensor (N.S.) 40, 173 (1983).

[8] E.G. Asanov, G.S.; Kirnasov, On 1-form Finsler spaces, Rep. Math. Phys. 19 (1984).

[9] J. Szilasi and L. Tamássy, Generalized Berwald spaces as affine deformations of Minkowski spaces, Rev. Roumaine Math. Pures Appl. 57, 165 (2012).

[10] Libing Huang, On the fundamental equations of homogeneous Finsler spaces, Differential Geom. Appl. 40, 187 (2015).

[11] N. Bartelmeß and V.S. Matveev, Monochromatic metrics are generalized Berwald, Diff. Geom. Appl. 58264 (2018).

[12] Z. I. Szabó, Positive definite Berwald spaces. Structure theorems on Berwald spaces, Tensor (N.S.) 35, 25 (1981).

[13] D. Bao, S.-S. Chern, and Z. Shen, An Introduction to Riemann-Finsler Geometry (Springer-Verlag, New York, 2000).

[14] E. Minguzzi, Raychaudhuri equation and singularity theorems in Finsler spacetimes, Class. Quantum Grav. 32, 185008 (2015), arXiv:1502.02313.

[15] E. Minguzzi, The connections of pseudo-Finsler spaces, Int. J. Geom. Meth. Mod. Phys. 11, 1460025 (2014), Erratum ibid 12 (2015) 1592001. arXiv:1405.0645.

[16] X. Cheng and Z. Shen, Finsler geometry, and approach via Randers spaces (Springer, Heidelberg, 2012).

[17] L. Berwald, Über Finslersche und Cartansche Geometrie II. Invarianten bei der Variation vielfacher Integrale und Parallelhyperflächen in Cartanschen Räumen, Compositio Math. 7, 141 (1939). 
[18] A. Moór, Ergänzung zu meiner Arbeit: "Über die Dualität von Finslerschen und Cartanschen Räumen.", Acta Math. 91, 187 (1954).

[19] G. Yu. Bogoslovsky and H. F. Goenner, On a possibility of phase transitions in the geometric structure of space-time, Physics Letters A 244, 222 (1998).

[20] G. Yu. Bogoslovsky and H. F. Goenner, Finslerian spaces possessing local relativistic symmetry, Gen. Relativ. Gravit. 31, 1565 (1999).

[21] G. Yu. Bogoslovsky, A special-relativistic theory of the locally anisotropic space-time. I: The metric and group of motions of the anisotropie space of events, Il Nuovo Cimento 40 B, 99 (1977).

[22] G. Yu. Bogoslovsky, A viable model of locally anisotropic space-time and the Finslerian generalization of the relativity theory, Fortschr. Phys. 42, 143 (1994).

[23] E. Minguzzi, Affine sphere spacetimes which satisfy the relativity principle, Phys. Rev. D 95, 024019 (2017), arXiv:1702.06745.

[24] A. Fuster and C. Pabst, Finsler pp-waves, Phys. Rev. D 94, 104072 (2016).

[25] J. I. Horváth, A geometrical model for the unified theory of physical fields, Phys. Rev. 80, 901 (1950).

[26] S. F. Rutz, A Finsler generalisation of Einstein's vacuum field equations, Gen. Relativ. Gravit. 25, 1139 (1993).

[27] J. M. Martín-García, xAct: efficient tensor computer algebra, http://www.xact.es.

[28] A. Bejancu and H. R. Farran, Geometry of pseudo-Finsler submanifolds (Kluwer Academic Publishers, Dordrecht, 2000).

[29] Z. Shen, Lectures on Finsler geometry (World Scientific, Singapore, 2001).

[30] J. Szilasi, R. L. Lovas, and D. Cs. Kertesz, Connections, sprays and Finsler structures (World Scientific, London, 2014).

[31] E. Minguzzi, Affine sphere relativity, Commun. Math. Phys. 350, 749 (2017), arXiv:1702.06739.

[32] Zejun $\mathrm{Hu}$, Haizhong Li, and Luc Vrancken, Locally strongly convex affine hypersurfaces with parallel cubic form, J. Differential Geom. 87, 239 (2011).

[33] R. Hildebrand, Centro-affine hypersurface immersions with parallel cubic form, Beiträge zur Algebra und Geometrie / Contributions to Algebra and Geometry 56, 593 (2015).

[34] L. D. Landau and E. M. Lifshitz, The Classical Theory of Fields (AddisonWesley Publishing Company, Reading, 1962). 
[35] A. G. Cohen and S. L. Glashow, Very special relativity, Phys. Rev. Lett. 97, 021601 (2006).

[36] G. W. Gibbons, J. Gomis, and C. N. Pope, General Very Special Relativity is Finsler geometry, Phys. Rev. D 76, 081701 (2007). 